\title{
Article
}

\section{FAmily-Led RehabiliTaTion aftEr Stroke in INDia: the ATTEND pilot study}

Pandian, Jeyaraj D, Felix, Cynthia, Kaur, Paramdeep, Sharma, Deepika, Julia, Lizzie, Toor, Gagan, Arora, Rajni, Gandhi, Dorcas B.C., Verma, Shweta J, Anderson, Craig S, Langhorne, Peter, Murthy, G.V.S, Hackett, Maree, Maulik, Pallab K, Alim, Mohammed, Harvey, Lisa A, Jan, Stephen, Walker, Marion, Forster, Anne and Lindley, Richard

Available at http://clok.uclan.ac.uk/11986/

Pandian, Jeyaraj D, Felix, Cynthia, Kaur, Paramdeep, Sharma, Deepika, Julia, Lizzie, Toor, Gagan, Arora, Rajni, Gandhi, Dorcas B.C., Verma, Shweta J et al (2015) FAmily-Led RehabiliTaTion aftEr Stroke in INDia: the ATTEND pilot study. international Journal of Stroke, 10 (4). pp. 609-614. ISSN 1747-4930

It is advisable to refer to the publisher's version if you intend to cite from the work. http://dx.doi.org/10.1111/ijs.12475

For more information about UCLan's research in this area go to http://www.uclan.ac.uk/researchgroups/ and search for <name of research Group>.

For information about Research generally at UCLan please go to http://www.uclan.ac.uk/research/

All outputs in CLoK are protected by Intellectual Property Rights law, including Copyright law. Copyright, IPR and Moral Rights for the works on this site are retained by the individual authors and/or other copyright owners. Terms and conditions for use of this material are defined in the policies page.

\section{CLoK}

Central Lancashire online Knowledge www.clok.uclan.ac.uk

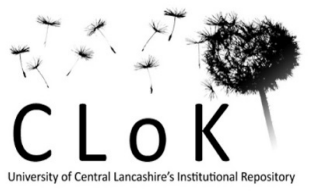




\section{FAmily-Led RehabiliTaTion aftEr stroke in INDia: The ATTEND Pilot Study}

Cover title: The ATTEND Pilot Study

Jeyaraj D Pandian DM FRACP, Cynthia Felix MD, Paramdeep Kaur PhD, Deepika Sharma BPT, Lizzie Julia MSc, Gagan Toor BPT, Rajni Arora MPT, Dorcas BC Gandhi MPT, Shweta J Verma MSc CR, Craig S Anderson MD PhD, Peter Langhorne MD PhD, G.V.S. Murthy MD MSc, Maree L Hackett PhD, Pallab K Maulik MD PhD MSc, Mohammed Alim M Pharm, Lisa A Harvey PhD, Stephen Jan PhD, Marion Walker PhD, Anne Forster PhD and Richard Lindley MD FRACP

\section{Author Affiliations}

From the Stroke Unit, Department of Neurology Christian Medical College, Ludhiana, Punjab, India (JDP, CF, PK, DS, LJ, SJV)

Department of Physiotherapy, Christian Medical College, Ludhiana, Punjab, India (RA, DBCG)

The George Institute for Global Health, University of Sydney and Royal Prince Alfred Hospital, Australia (CA)

The George Institute for Global Health, University of Sydney (MLH, SJ, RL)

University of Glasgow, UK (PL)

Indian Institute of Public Health Hyderabad, Public Health Foundation of India, India (GVSM)

University of Central Lancashire, UK (MLH)

The George Institute for Global Health, India (PKM, MA) 
The George Institute for Global Health, Oxford University, UK (PKM)

Sydney Medical School/Northern, University of Sydney, Australia (LAH)

University of Nottingham, UK (MW)

University of Leeds, UK (AF)

\section{Corresponding Author}

Dr. Jeyaraj D Pandian MD DM FRACP

Professor and Head, Department of Neurology

Deputy Director (Research and Development)

Christian Medical College and Hospital, Ludhiana, Punjab 141008, India

Phone: 91-9915784750

Fax no. : 91-0161-2220850

E-mail: jeyarajpandian@hotmail.com

Key words: Stroke, Rehabilitation, Therapy, Early Supported Discharge, Home-based Care, Caregiver, Quality of Life, Developing countries

Word count: 3,597

\section{Tables}

Table 1: The baseline demographic details in both the groups $(\mathrm{n}=104)$

Table 2: Primary outcome measure (modified Rankin Scale)

Table 3: Secondary outcomes (WHOQOL, ADL, HADS, CBS and EQ-5D) 
Table 4: Direct medical cost at the time of admission $(n=104)$

\section{Figures}

Figure 1: CONSORT flow diagram

Figure 2: Feasibility issues 


\begin{abstract}
Background and purpose: The aim of this pilot study was to determine the feasibility of a multicenter, randomized, controlled trial in India of a family-led trained caregiver-delivered home-based rehabilitation intervention versus routine care.
\end{abstract}

Methods: A prospective, randomized (within 7 days of hospital admission), blinded outcome assessor, controlled trial of structured home-based rehabilitation delivered by trained and protocol-guided family caregivers (intervention) versus routine care alone (control), in patients with residual disability. Key feasibility measures were recruitment, acceptance and adherence to assessment procedures and follow up of participants over 6 months.

Results: A total of 104 patients from the stroke unit at Christian Medical College, Ludhiana were recruited over 9 months. Recruitment was feasible and accepted by patients and their carers. Important observations were made regarding potential unblinding of the participants, contamination of therapy between the randomized groups, organization of home visits, and resources required for a multicenter study.

Conclusion: The pilot study established the feasibility of conducting a large scale study of family-led, trained caregiver-delivered, home-based stroke rehabilitation in a low resource setting. The main phase of the trial 'ATTEND' is currently underway in over 10 centers in India.

Clinical trial registration-URL: http://www.clinicaltrials.gov

Unique identifier: NCTO2123875 


\section{Introduction}

The Global Burden of Disease project 2010 indicates that most of the global burden of stroke is on low and middle income countries (LMICs). ${ }^{1}$ In India, for example, the annual rate of stroke ranges from 135 to 145 per $100,000,,^{2-4}$ and a high proportion of survivors are left with disability without sufficient access to rehabilitation. ${ }^{2-4}$

While evidence-based treatments for stroke such as acute stroke unit (SU) care ${ }^{5}, 6$ are established worldwide, most Indian SUs are located in private hospitals in urban areas. A significant proportion of the cost of care is borne by the patients who generally have limited access to health insurance. ${ }^{7}$ The average cost for stroke in a private hospital in India is estimated at rupees (INR) 80,612 (USD 1520) for the first 6 months post-stroke, which is equivalent to the average per capita income in India, thus making acute care and rehabilitation unaffordable to much of the population affected by stroke. ${ }^{8}$

Innovative models of stroke care in LMICs may capitalize on the extended family system that provides much of the care for the sick or disabled. These attempt to train and empower the caregivers as the 'virtual multidisciplinary team', to deliver home-based rehabilitation, potentially improving outcomes while reducing health care costs. Thus the FAmily-Led RehabiliTaTion aftEr stroke in INDia (ATTEND) trial was developed as a modified Early Supported Discharge strategy to test the effectiveness of this concept.

In order to ascertain the feasibility of undertaking such a large-scale investigation across multiple sites in India, we undertook a pilot phase to establish the potential for recruitment, fidelity of the intervention package and to ensure that the procedures for assessment, instruction and follow up were methodologically sound and feasible.

\section{Methods}




\section{Design}

A prospective, randomized, open blinded outcome assessor, controlled trial (PROBE) design was used to assess trained family-led caregiver-delivered, home-based stroke rehabilitation compared to routine care. The study site for the pilot phase was the Stroke Unit of the Department of Neurology at Christian Medical College and Hospital in Ludhiana, India.

Patients were included if they were aged $\geq 18$ years with residual disability (defined as requiring help from another person for everyday activities), within one month of a clinically definite acute stroke of any pathological type except subarachnoid hemorrhage. Patients were excluded if they were assessed as being at a high probability of death within the next 6 months, or were unable to identify a suitable family-nominated caregiver for training and subsequent delivery of care. All patients (or their authorized representative) provided consent and the study was approved by the ethics committee of Christian Medical College and Hospital, Ludhiana.

\section{Procedures}

Eligible patients were randomized within 7 days of hospital admission, using random allocation software (RALOC) version 1.0.0 (developed by M. Saghaei, Isfahan University of Medical Sciences, Iran). This list was generated by a biostatistician and conveyed by telephone to the trial physiotherapist.

Patients allocated to the intervention arm had their family nominated caregiver trained by a trial physiotherapist, using a structured assessment (cognition, language, function and mobility) and recommended rehabilitation package. Components of the evidence based intervention package included: (i) information on stroke recovery trajectory, risk, identification and management of low mood and the importance of repeated practice of task 
specific activities, (ii) joint goal setting with patient, nominated family caregiver and therapist (reviewed with the therapist as patient progresses and new goals set), (iii) positioning, transfers and mobility, (iv) task orientated training (particularly walking, upper-limb and selfcare tasks) and (v) discharge planning. The local team developed a culturally appropriate, simple, pictorial 'manual' covering key exercises relevant to activities of daily living. In addition to the manual, training exercises were also chosen from the website http://www.physiotherapyexercises.com or as determined best for the patient by the therapist, all adhering to the intervention package.

The trial therapist was trained in delivering the intervention package to the patient caregiver. The caregiver training was to commence in the hospital for approximately 60 minutes per day for about 3 days (with the intention of accelerating the patient's hospital discharge when it was safe to do so). Afterwards, the caregiver would continue the intervention when the patient was discharged home. The trial therapist was able to be contacted through telephone for support and guidance over the next 3 months.

Patients randomized to receive routine care were free to access rehabilitation services provided on an in-or-out- patient basis after discharge from hospital but caregivers were not provided with trial-specific training.

The primary feasibility measures were recruitment, acceptance of intervention and assessments, and loss to follow-up. The primary clinical outcome of the trial was good functional recovery defined by scores $0-2$ on the mRS (modified Rankin Scale) ${ }^{9}$ at 3 and 6 months.

Secondary clinical outcomes included a battery of planned measures to be used in the main phase of the trial: simple validated recovery and dependency questions, ${ }^{10}$ WHO Quality of Life (WHOQOL)- BREF, ${ }^{11,12}$ Nottingham Extended Activities of Daily Living (NEADL), ${ }^{13}$ 
Hospital Anxiety and Depression Scale (HADS), ${ }^{14}$ Caregiver Burden Scale (CBS) ${ }^{15}$ and Euro QoL (EQ-5D-3L) ${ }^{16,17}$ (all at 3 and 6 months) and direct medical costs associated with health care utilization. Reported here are the combined results from both treatment groups to illustrate the range of outcomes for the included patients.

Follow-up assessment and documentation of outcomes were undertaken by face-to-face interview, either at home (for a small number of patients) or in a clinic at the hospital, at 3 and 6 months. These assessments were done by a psychologist who was blinded to the treatment allocation.

\section{Statistical analysis}

The data by treatment allocation from the participants presented in this pilot study will contribute to the planned meta-analysis when data for the main trial is published and therefore primary and secondary outcomes divided by group and between-group differences are not presented here. Instead, data were analyzed descriptively [with frequencies, means, medians and interquartile ranges] using SPSS version 21.0 (Armonk, NY: IBM Corp 2013).

\section{Results}

A total of 379 patients were assessed for eligibility and 104 patients were recruited between December 2011 and September 2012. Fifty patients were allocated to the intervention group, and fifty four to the control group. Loss to follow-up at 6 months was 15 participants; 89 patients (intervention group: 44; control group: 45) completed the trial (Figure 1). Other feasibility issues are shown in Figure 2.

The baseline demographic details are shown in Table 1 . The grouped primary and secondary outcome measures are shown in Table 2 and 3 respectively. Out of the 90 patients followed up at 90 days, 26 (29\%) patients had a good outcome (mRS 0-2) and 64 (71\%) a poor 
outcome (mRS 3-6). At the $6^{\text {th }}$ month of follow-up (n=89), 35 (39\%) had a good outcome and 54 (61\%) had a poor outcome. The median direct medical cost was USD 405 (interquartile range: USD 260- USD 882) and the highest charges were incurred for 'hospital stay charges', the median for which was USD 162 (Table 4).

\section{Discussion}

The pilot trial demonstrated excellent recruitment at the study site. In a span of 9 months, 104 patients were recruited. The recruitment was achieved using just one full-time trial staff (in addition to the treating neurologist).

Unlike in many drug trials where patients may often decline randomization due to fear of side effects, all of the medically stable patients from those screened for eligibility, agreed to be randomized.

The intervention, being a simple one, is easily understood by the lay persons in India, including many who are uneducated and illiterate, and this probably spurred patient and caregiver interest which was sustained over time. The therapist was also likely to have established a good rapport with the patients' families, which we hope facilitates tapping the unrealized human resources within the family for cost-effective stroke rehabilitation.

We note that only $39 \%$ of the participants were independent (mRS: $0-2$ ) at six months, confirming the poor long-term outcome of our selected trial population. These data, together with the other summary results (Tables 1-4) highlight the potential for improving post stroke rehabilitation in India using the family caregiver.

But we also learnt some valuable lessons from the pilot study, as we prepared to recruit for the main study. 
One main issue, as expected, was contamination between groups. The trial therapist also being the routine hospital physiotherapist for the unit naturally caused contamination. Also, the trial intervention manual was given to the intervention patient in the hospital that aroused interest from other patients, also leading to intervention contamination. For the main study, since there is more funding key changes to the logistics of running the trial included the decision to have dedicated trial staff for recruiting and delivering the intervention. This strategy was adopted to avoid therapists treating both control and experimental participants. Trial standard operating procedures were also developed to further reduce the risk of contamination e.g. the intervention to experimental participations will be provided in a separate area or behind curtains, the trial manual will only be provided at the first home visit by the trial therapist, the routine hospital physiotherapists will not participate in any trial procedures or training.

Fifteen patients were lost to follow-up (which included two who refused follow-up); the majority was due to a wrong contact number, frequent changes in mobile phone numbers and changes in residential locations. For the main study, we sought to overcome this by gathering multiple phone numbers and addresses of patients and relatives and more funding allocation for the follow-up study staff to travel in search of trial subjects.

The questionnaires, including the multiple complex tools used in the pilot study were overall found to be appropriate for our trial population. But we further refined and redesigned the questionnaires for the main study to tightly match the study protocol. They were translated to vernacular languages of each site, in addition to English, for easy participant understanding.

For the multicenter roll-out of the main trial, we have developed a more detailed intervention manual capturing the important components of the intervention (assessment, goal setting, and training activities) with a greater emphasis on evidence-based functional task activities. The 
main trial therapists would be trained by a team of experts in delivering the intervention package uniformly across sites.

We would be gathering study logs for the main study to determine the fidelity of the intervention- assessing the quality and quantity of intervention delivered. We would also be using methods to determine the acceptability of the outcome measures to the participants. We would, in addition, assess levels of unblinding amongst the main study outcome assessors by evaluating their experience.

The pilot study, thus, gave us the rich training ground to test a complex behavioral intervention which has never before been done in India- family-led stroke rehabilitation. In LMICs, ongoing face-to-face physiotherapy is rarely available except to an elite few. There are not many out-patient therapy departments in the remote parts of the country or in many public (government-run) hospitals, and most families cannot afford transporting the sick stroke patient to receive out-patient therapy, all of which cause high levels of disability in stroke patients. Traditional long-term rehabilitation costs often limit the chronic recovery phase of stroke and low-cost alternatives seem to be the need of the hour.

The lessons learnt from the pilot trial have formed the platform for the design of the main trial (ATTEND), which commenced recruitment in 2014 in more than 10 centers across India.

Our feasibility data also strengthened the funding application for the main study that was granted by the Australian National Health and Medical Research Council at the end of 2012.

\section{Conclusion}

The pilot study highlighted the logistical issues that need to be considered for conducting a larger trial. The pilot also showed that it was feasible to conduct a trial (ATTEND) using an 
intervention of an empowered caregiver to give home-based rehabilitation after stroke in India, which if positive has the potential to have a major impact in the delivery of costeffective stroke rehabilitation across the world. Such a home-based program may be useful even in developed countries, by tapping the underutilized enthusiasm of a loved one in caring for a sick family member, especially in a condition like stroke, where a few months of continuous rehabilitation can potentially minimize morbidity.

\section{Author contributions:}

JDP: Conception, design, interpretation of data, supervision of manuscript draft and revision of the manuscript with critical contribution for intellectual content, giving final approval of the version to be published

CF: Conception, design, interpretation of data, drafting of all versions of the manuscript, revision of the manuscript with critical contribution for intellectual content

PK: Analysis and interpretation of data

DS: Conducted study intervention

LJ: Conducted study follow-up

GT: Worked on study manual

RA: Contributed to intervention methodology

DBCG: Contributed to design and partial drafting of manuscript original version

SJV: Contributed to design and partial drafting of manuscript original version

CSA: Interpretation of data and revision of the manuscript for intellectual content

PL: Interpretation of data and revision of the manuscript for intellectual content 
GVSM: Interpretation of data and revision of the manuscript for intellectual content

MLH: Interpretation of data and revision of the manuscript for intellectual content

PKM: Interpretation of data and revision of the manuscript for intellectual content

MA: Interpretation of data and revision of the manuscript for intellectual content

LAH: Interpretation of data and revision of the manuscript for intellectual content

SJ: Interpretation of data and revision of the manuscript for intellectual content

MW: Interpretation of data and revision of the manuscript for intellectual content

AF: Interpretation of data and revision of the manuscript for intellectual content

RL: Conception, design and interpretation of data, and critical revision of the manuscript for intellectual content

All authors read and approved the final manuscript.

\section{Acknowledgements}

We thank the patients and families who participated in this study. We acknowledge Dr. Santhosh Mathangi MD, for his support in designing the intervention manual.

During the completion of this work, Maree Hackett was in receipt of a National Health and Medical Research Council (NHMRC) Career Development Fellowship and a National Heart Foundation Future Leader Fellowship 100034; Craig Anderson held a Senior Principal Research Fellowship of the NHMRC. These funding bodies had no role in the conduct or reporting of the trial.

\section{Funding}


Australian NHMRC Program (571281) and Project (1045391) grants and The George Institute for Global Health, University of Sydney, Australia

\section{Conflict of interest}

None

\section{References}

1. Feigin VL, Forouzanfar MH, Krishnamurthi R, et al. Global and regional burden of stroke during 1990-2010: findings from the global burden of disease study 2010. Lancet 2014;383:245-254.

2. Das SK, Banerjee TK, Biswas A, et al. Prospective community-based study of stroke in Kolkata, India. Stroke 2007;38:905-910.

3. Dalal PM, Malik S, Bhattacharjee M, et al. Population-based stroke survey in Mumbai, India: incidence and 28-day case fatality. Neuroepidemiology 2008;31:254-261.

4. Sridharan SE, Unnikrishnan JP, Sukumaran S, et al. Incidence, types, risk factors, and outcome of stroke in a developing country, The Trivandrum Stroke Registry. Stroke 2009;40:1212-1218.

5. Langhorne P, Sandercock P, Prasad K. Evidence-based practice for stroke. Lancet Neurol 2009;8:308-309.

6. Gilligan AK, Thrift AG, Sturm JW, Dewey HM, Macdonell RA, Donnan GA. Stroke units, tissue plasminogen activator, aspirin and neuroprotection: which stroke intervention could provide the best community benefit? Cerebrovasc Dis 2005;20:239244.

7. Pandian JD, Sudhan P. Stroke epidemiology and stroke care services in India. J Stroke 2013;15: 128-134. 
8. Kwatra G, Kaur P, Toor G, et al. Cost of stroke from a tertiary center in northwest India. Neurol India 2013;61:627-632.

9. Bonita R, Beaglehole R. Recovery of motor function after stroke. Stroke 1988;19:14971500.

10. Lindley RI, Waddell F, Livingstone M, et al. Can simple questions assess outcome after stroke? Cerebrovascular Diseases 1994;4:314-324.

11. WHOQOL GROUP. The world health organization quality of life assessment (WHOQOL): Position paper from the world health organization. SocSci Med 1995; 41:1403-1409.

12. The WHOQOL group. Development of the world health organization WHOQOL-BREF quality of life assessment. Psychol Med 1998b;28:551-558.

13. Nouri FM, Lincoln N. An extended ADL scale for use with stroke patients. Clin Rehabil 1987;1:301-305.

14. Zigmond AS, Snaith RP. The hospital anxiety and depression scale. ActaPsychiatrScand 1983;67:361-370.

15. Elmstahl S MB, Annerstedt L. Caregiver's Burden of Patients 3 Years after Stroke Assessed by a Novel Caregiver Burden Scale. Arch Phys Med Rehabil 1996;77:177182.

16. The EuroQol Group. EuroQol-a new facility for the measurement of health-related quality of life. Health Policy 1990;16(3):199-208.

17. Brooks R. EuroQol: the current state of play. Health Policy 1996;37(1):53-72. 
Table 1: The baseline demographic details in both the groups $(n=104)$

\begin{tabular}{|c|c|}
\hline Variables & Number (\%) \\
\hline Age (Mean \pm SD) ${ }^{*}$ & $60 \pm 13$ years \\
\hline \multicolumn{2}{|l|}{ Gender } \\
\hline Men & 61 (59) \\
\hline \multicolumn{2}{|l|}{ Marital status } \\
\hline Married & $101(98)$ \\
\hline Unmarried & $2(2)$ \\
\hline \multicolumn{2}{|l|}{ Caregiver } \\
\hline Spouse & $64(63)$ \\
\hline Other relative & 37 (37) \\
\hline \multicolumn{2}{|l|}{ Education } \\
\hline Lower $^{\dagger}$ & $25(24)$ \\
\hline Upper $^{\ddagger}$ & 77 (76) \\
\hline \multicolumn{2}{|l|}{ Living situation } \\
\hline Independent & $101(97)$ \\
\hline \multicolumn{2}{|l|}{ Annual income } \\
\hline \multicolumn{2}{|l|}{$(\mathrm{INR})^{\S}$} \\
\hline$<14,999$ & $56(54)$ \\
\hline $15,000-29,999$ & $35(34)$ \\
\hline $30,000-1,00,000$ & $13(12)$ \\
\hline \multicolumn{2}{|l|}{ Family } \\
\hline Joint & $73(70)$ \\
\hline Nuclear & $31(30)$ \\
\hline
\end{tabular}


*standard deviation, ${ }^{\dagger}$ less than primary school completed, ${ }^{\ddagger}$ completed secondary school and beyond, ${ }^{\S}$ Indian rupees 
Table 2: Primary outcome measure (modified Rankin Scale)

\begin{tabular}{ll}
\hline Variables & Number (\%) \\
\hline mRS $^{*}$ at 3 months follow-up & $\mathbf{n = 9 0}$ \\
Good outcome & \\
Poor outcome & \\
mRS $^{*}$ at $\mathbf{6}$ months follow-up $^{+}$ & $26(29)$ \\
Good outcome $^{\dagger}$ & $64(71)$ \\
Poor outcome $^{\ddagger}$ & $35(39)$ \\
\hline
\end{tabular}

${ }^{*}$ modified Rankin Scale, ${ }^{\dagger} \mathrm{mRS}:$ 0-2, ${ }^{\ddagger} \mathrm{mRS}: 3-6$ 
Table 3: Secondary outcomes (WHOQOL, ADL, HADS, CBS and EQ-5D)

\begin{tabular}{ll} 
Variables & Median (IQR) \\
\hline
\end{tabular}

At 3 months follow-up $\quad n=75$

WHO QOL ${ }^{\dagger}$

Physical domain

$50(38-63)$

Psychological domain

$50(38-56)$

Social domain

$56(50-75)$

Environment domain

$56(44-64)$

$\mathbf{A D L}^{\ddagger}$

$15(5-19)$

HADS $^{\S}$

Number (\%)

Anxiety

38 (51)

Depression

$25(34)$

CBS scale

Burden

35 (47)

EQ-5D

Mobility

Problems

$41(55)$

Self-care

Problems

$45(61)$

Normal Activities

Problems

47 (63)

Pain/Discomfort

Problems

51 (69)

Anxiety/Depression 
Problems

At 6 months follow-up

WHO QOL

Physical domain

Psychological domain

Social domain

Environment domain

ADL

HADS

Anxiety

Depression

CBS scale

Burden

EQ-5D

Mobility

Problems

Self-care

Problems

Normal Activities

Problems

\section{Pain/Discomfort}

Problems

Anxiety/Depression

Problems
$34(50)$

$31(46)$

$51(69)$

$n=69$

Median (IQR)*

$56(50-69)$

$56(50-69)$

$75(56-81)$

$63(50-75)$

$18(16-21)$

Number (\%)

$27(40)$

$19(28)$$$
31 \text { (46) }
$$

$31(46)$

$41(60)$

42 (62)

${ }^{*}$ interquartile range, ${ }^{\dagger}$ WHO Quality of Life, ${ }^{\ddagger}$ Nottingham extended activities of daily living, ${ }^{\S}$ Hospital Anxiety and Depression Scale, 'Caregiver Burden Scale 
Table 4: Direct medical cost at the time of admission $(n=104)$

\section{Variables}

\section{Median (IQR)*}

INR $^{\dagger} \quad$ USD

$\begin{array}{lll}\text { Total Direct Medical Cost } & 24312(15618-52889) & 405(260-882) \\ \text { Admission charges } & 638(616-694) & 11(10-12) \\ \text { Hospital stay charges } & 9700(5755-14590) & 162(96-243) \\ \text { Rehabilitation }{ }^{\ddagger} \text { Charges } & 980(540-1755) & 16(9-29) \\ \text { Laboratory Charges } & 5750(3910-6730) & 96(65-112) \\ \text { Imaging Charges } & 6600(3230-8545) & 110(54-142) \\ \text { Drug Charges } & 6089(2670-18976) & 101(45-316)\end{array}$

${ }^{*}$ interquartile range, ${ }^{\dagger}$ Indian rupees, ${ }^{\ddagger}$ included physiotherapy and occupational therapy 